Published in final edited form as:

J Am Acad Child Adolesc Psychiatry. 2012 November ; 51(11): 1150-1159. doi:10.1016/j.jaac.

2012.08.018.

\title{
Early Behavioral Intervention Is Associated With Normalized Brain Activity in Young Children With Autism
}

\author{
Geraldine Dawson, Ph.D., Emily J.H. Jones, Ph.D., Kristen Merkle, B.S., Kaitlin Venema, \\ B.S., Rachel Lowy, B.S., Susan Faja, Ph.D., Dana Kamara, B.S., Michael Murias, Ph.D., \\ Jessica Greenson, Ph.D., Jamie Winter, Ph.D., Milani Smith, Ph.D., Sally J. Rogers, Ph.D., \\ and Sara J. Webb, Ph.D. \\ Dr. Dawson is with the University of North Carolina at Chapel Hill, the University of Washington \\ (UW), and Autism Speaks. Drs. Jones, Faja, Murias, Greenson, Winter, Smith, and Webb, and \\ Ms. Venema, Ms. Lowy, and Ms. Kamara are with the Center on Human Development and \\ Disability (CHDD) at UW. Ms. Merkle is with Vanderbilt University. Dr. Rogers is with the \\ University of Davis MIND Institute
}

\begin{abstract}
Objective-A previously published randomized clinical trial indicated that a developmental behavioral intervention, the Early Start Denver Model (ESDM), resulted in gains in IQ, language, and adaptive behavior of children with autism spectrum disorder. This report describes a secondary outcome measurement from this trial, EEG activity.
\end{abstract}

\begin{abstract}
Method-Forty-eight 18- to 30-month-old children with autism spectrum disorder were randomized to receive the ESDM or referral to community intervention for 2 years. After the intervention (age 48 to 77 months), EEG activity (event-related potentials and spectral power) was measured during the presentation of faces versus objects. Age-matched typical children were also assessed.
\end{abstract}

\begin{abstract}
Results-The ESDM group exhibited greater improvements in autism symptoms, IQ, language, and adaptive and social behaviors than the community intervention group. The ESDM group and typical children showed a shorter Nc latency and increased cortical activation (decreased a power and increased $\theta$ power) when viewing faces, whereas the community intervention group showed the opposite pattern (shorter latency event-related potential [ERP] and greater cortical activation when viewing objects). Greater cortical activation while viewing faces was associated with improved social behavior.
\end{abstract}

Conclusions-This was the first trial to demonstrate that early behavioral intervention is associated with normalized patterns of brain activity, which is associated with improvements in social behavior, in young children with autism spectrum disorder.

\section{Keywords}

autism; early behavioral intervention; Early Start Denver Model; event-related potentials; brain activity

\footnotetext{
(C) 2012 American Academy of Child and Adolescent Psychiatry

Correspondence to Geraldine Dawson, Ph.D., Department of Psychiatry, School of Medicine, 4120 Bioinformatics Building, \#3366, University of North Carolina, Chapel Hill, NC 27599; gdawson@autismspeaks.org.

Disclosure: Drs. Dawson and Rogers are authors of the book, Early Start Denver Model for Young Children with Autism (Guilford Press), from which they receive royalties. Dr. Murias is a minority shareholder in Electrical Geodesics, Inc. Drs. Jones, Faja, Greenson, Winter, Smith, and Webb and Ms. Merkle, Ms. Venema, Ms. Lowy, and Ms. Kamara report no biomedical financial interests or potential conflicts of interest.
} 
Autism spectrum disorder (ASD) is characterized by impairments in social interaction and communication and stereotyped and repetitive behaviors. With a prevalence of one per $88,{ }^{1}$ autism represents a serious public health challenge. Autism can be diagnosed by 18 to 24 months of age, ${ }^{2}$ and diagnostic assessments for 12- to 18 -month-olds are being developed. ${ }^{3}$ Given that the American Academy of Pediatrics has recommended that 18- and 24-monthold children be screened for ASD, ${ }^{4}$ it is important that effective early-intervention programs be available.

A randomized controlled trial was conducted to evaluate the efficacy of the Early Start Denver Model (ESDM), 5 a comprehensive developmental behavioral intervention, for improving the outcomes of toddlers with ASD. Compared with children who received the commonly available community intervention (CI), children who received ESDM for 2 years showed significant improvements in IQ, language, adaptive behavior, and autism diagnosis. The ESDM group on average improved 17.5 standard score points (standard deviation [SD] 15 points) compared with 7.0 points in the comparison group. This was the first randomized controlled trial to demonstrate the efficacy of a comprehensive behavioral intervention for toddlers with ASD.

Of interest is whether and how early intervention alters the trajectory of brain development. The development of social and language brain circuitry-its acquisition, organization, and function-results from the interaction between a young child and his or her social environment. During reciprocal social interactions, engagement with a social partner facilitates the cortical specialization of perceptual and representation systems for social and linguistic information. Therefore, the early lack of social engagement associated with ASD could have negative secondary effects on behavioral and brain development. Early intervention that is designed to enhance social attention and affective and social engagement, thereby promoting the development of cognitive and language skills, may serve to alter a child's brain development toward a more normal developmental trajectory. ${ }^{6}$ To evaluate this hypothesis, the authors measured EEG activity while viewing social (faces) and nonsocial (toys) stimuli in young children with ASD who participated in a randomized clinical trial of ESDM, an early intervention that focuses on social motivation and engagement.

\section{Early Patterns of EEG Activity in ASD}

Research has documented atypical patterns of EEG activity in young children with ASD in response to face stimuli. ${ }^{7-9}$ This atypical response is characterized by delays in the development of an attention-related event-related potential (ERP) component, the Nc, and a face-specific perceptual processing component, the N290 (and later in development, the N170). ${ }^{8,9}$ The Nc component, the source of which is the prefrontal cortex and anterior cingulate cortex, reflects attention engagement with a stimulus. ${ }^{10}$ The N170 reflects earlystage perceptual processing of faces, the source of which is the temporal/fusiform region. ${ }^{11,12}$

Event-related decreases in a power and increases in $\theta$ power reflect higher levels of cortical activation and have been associated with increased attention and cognitive processing of a stimulus (for reviews, see Başar et al. ${ }^{13}$ and Klimesch ${ }^{14}$ ). The a oscillations have been shown to emerge from interactions with the thalamocortical network and have been demonstrated to be present in subcortical areas, including the hippocampal region. ${ }^{15}$ The a oscillation results from a reciprocal interplay between excitatory and inhibitory neurons and is influenced by cholinergic, serotonergic, and glutamatergic machanisms. ${ }^{15}$ The a activity increases during a relaxed state and decreases during active stimulus processing. 
The $\theta$ oscillations are especially prominent in the hippocampal region, are influenced by the interaction between glutamatergic and $\boldsymbol{\gamma}$-aminobutyric acidergic neurons, and are a proposed mechanism for synaptic plasticity. ${ }^{15}$ It is well established that $\theta$ activity is involved in memory-related functions and is increased during tasks requiring working memory and focused attention. Individuals with Asperger syndrome show relatively lower levels of $\theta$ power when viewing emotional faces compared with typical individuals. ${ }^{16}$ Typical infants have demonstrated sharp increases in $\theta$ power when engaged in a peek-a-boo game compared with viewing objects. ${ }^{17}$ Infants and preschool-age children have shown increases in $\theta$ spectral power when viewing social stimuli and toys compared with a baseline condition. ${ }^{18}$

The present article reports data on EEG activity collected during viewing of social (faces) and nonsocial (toys) stimuli in children with ASD who participated in a randomized clinical trial of the ESDM, a developmental behavioral intervention designed to improve cognitive, language, and social functioning, which focuses on increasing social attention and engagement and enhancing the social-affective relationship. At the outcome, the authors examined two aspects of information processing derived from the EEG: early-stage perceptual processing of faces (N170 ERP) and allocation of attention or engagement with social versus nonsocial stimuli. The latter was reflected in the ERP Nc component and levels of cortical activation (EEG $a$ and $\theta$ powers). The authors predicted that children with typical development and children who received the ESDM intervention would exhibit shorter latency N170 and Nc responses and higher levels of cortical activation (decreased a power and increased $\theta$ power) when viewing faces compared with toys, whereas children with ASD receiving intervention in the community would show the reverse pattern (faster ERP $\mathrm{N} 170$ and $\mathrm{Nc}$ responses and greater cortical activation when viewing objects). The authors also examined the relation between EEG activity and behavior outcomes.

\section{Method}

\section{Participants}

The study was approved by the University of Washington institutional review board; informed consent was obtained from the participants' parents. Forty-eight participants with ASD were 18 to 30 months of age at entry to the study and had a diagnosis of an autistic disorder or a pervasive developmental disorder (PDD) not otherwise specified. Participants with ASD were recruited through pediatric practices, Birth-to-Three centers, preschools, hospitals, and state and local autism organizations. The exclusion criteria included a neurologic disorder of known genetic etiology, a significant sensory or motor impairment, major physical problems, seizures at the time of entry, use of psychoactive medications, a history of a serious head injury and/or neurologic disease, alcohol or drug exposure during the prenatal period, and a nonverbal IQ below 35 . The inclusion criteria included an age younger than 30 months at entry, meeting the criteria for an autistic disorder on the Toddler Autism Diagnostic Interview (ADI ${ }^{19}$ and for autism or ASD on the Autism Diagnostic Observation Schedule (ADOS) ${ }^{20}$ and a clinical diagnosis based on $D S M-I V$ criteria $^{21}$ using all available information, and residing within 30 minutes of the University of Washington. Detailed descriptions of the children at entry and primary outcomes are reported elsewhere. ${ }^{5}$

Typically developing children were matched by chronologic age to children with ASD at the time of EEG assessment. Their ages ranged from 48 to 63 months and they were recruited from the University of Washington Communications Studies Participant Pool. They had no first- or second-degree relatives with ASD and had standard scores from 80 to 125 (Vineland Adaptive Behavior Scales). There were no significant differences among groups in chronologic age or gender. 
The ethnicities of the participants were Asian (12.5\%), white (72.9\%), Latino (12.5\%), and multiracial $(14.6 \%)$; there were no differences among groups $\left(\chi^{2}=0.69, p=.41\right)$. The maleto-female ratio of participants with ASD was 3.5:1.

\section{Study Design}

At entry, children with ASD were randomized into two groups: ESDM, which received yearly assessments and offered 20 hours per week of intervention from trained clinicians, parent training, and parent delivery for more than 5 hours per week of the ESDM in addition to whatever community services the parents chose or CI, which received yearly assessments with recommendations and referrals for intervention with community providers in the greater Seattle region. The actual mean hours per week of intervention delivered by professionals were 20.4 in the ESDM group and 18.4 in the CI group.

Early Start Denver Model-Trained therapists offered the intervention for 2 hours, twice a day, 5 days a week, for 2 years. ESDM therapists were supervised by a Ph.D.-level, highly experienced lead therapist. Therapists were required to demonstrate and maintain a fidelity of $85 \%$ of maximum scores on the fidelity instrument. An intervention manual and curriculum were used. One or both parents were provided with ongoing parent training during semimonthly meetings, and parents used the ESDM strategies during daily activities. The ESDM is a developmentally informed treatment and curriculum that addresses all developmental domains, uses teaching strategies based on applied behavior analysis, and emphasizes interpersonal exchange and positive affect, social attention, and shared engagement. Children were trained in face recognition with individualized booklets of color photographs of the faces of four familiar people (e.g., mother, father, sibling, and therapist). There were no significant adverse events associated with the ESDM intervention.

Community Intervention-The CI group received comprehensive diagnostic evaluations, intervention recommendations, and community referrals at baseline and at each of the two annual follow-up assessments. Families were given resource manuals and reading materials at baseline and twice yearly. Children participated in interventions delivered by Birth-to-Three centers and individual providers delivering speech-language therapy, occupational therapy, and/or applied behavior analysis treatments. Retention rates in the intervention trial were $100 \%$ (ESDM) and $88 \%(\mathrm{CI})$.

\section{Randomization}

Participants were randomized into two groups based on the composite IQ at entry $(<55$ and 255 ) and gender to ensure comparable IQ and gender ratios between groups. Within each of these strata, randomization was conducted by using random permuted blocks of 4 . Refer to Dawson et al..$^{5}$ for a flowchart of the study design.

\section{Behavioral Outcome Measurements}

At outcome, when children with ASD were 48 to 54 months old, the following behavioral measurements were collected. The ADI-Revised ${ }^{18}$ is a parent interview assessing developmental history and autism symptoms. The ADOS, Western Psychological Services version, ${ }^{19}$ is a standardized observation that assesses $D S M-I V^{21}$ autism symptoms in social relatedness, communication, play, and repetitive behaviors. The Mullen Scales of Early Learning (MSEL) ${ }^{22}$ is a standardized developmental test for children from birth to 68 months of age; it has four subscales: Fine Motor, Visual Reception, Expressive Language, and Receptive Language. The Vineland Scales of Adaptive Behavior ${ }^{23}$ is a parent interview assessing social, communication, motor, and daily living skills. The PDD Behavioral 
Inventory (BI) ${ }^{24}$ is a parent-report questionnaire assessing social approach/withdrawal problems and receptive/expressive social communication abilities.

\section{EEG Assessment and Participant Attrition}

The EEG was assessed when children were 49 to 77 months old, on average 2.5 months (SD 0.9 month) after the behavioral assessment. Close to $60 \%$ of children with ASD provided artifact-free data, an attrition rate comparable to other EEG studies of children with ASD. Attrition was primarily due to an inability to comply with the procedure or excessive movement artifact. Artifact-free EEG data were provided by 15 children in the ESDM group (eight with a clinical diagnosis of an autistic disorder and seven with PDD not otherwise specified), 14 children in the CI group (12 with a clinical diagnosis of an autistic disorder and two with PDD not otherwise specified), and 17 typical children. Typical children had higher Vineland Scales of Adaptive Behavior scores than the ASD groups $\left(F_{1,44}>31.9, p<\right.$. 001) but did not differ in chronologic age (ASD, mean 54.1 months, SD 6.3 months; typical, mean 55.7 months, SD 4.5 months; $F_{1,44}=0.8, p=.4$ ). Children with artifact-free EEG data in the ESDM and CI groups did not differ in chronologic age (ESDM, mean 54.1 months, SD 4.9 months; CI, mean 54.1 months, SD 7.8 months; $F_{1,27}=0.00, p=.99$ ), baseline ADOS Social scores (ESDM, mean 10.3, SD 2.3; CI, mean 11.1, SD 2.7), ADOS Restricted and Repetitive Behaviors (ESDM, mean 2.6, SD 1.3; CI, mean 3.6, SD 2.0), MSEL Verbal IQ (ESDM, mean 45.3, SD 17.5; CI, mean 48.1, SD 21.2), and MSEL Nonverbal IQ (ESDM, mean 83.6, SD 13.3; CI, mean 79.2, SD 11.3; $F_{1,27}<2.5, p>.13$, for all comparisons). Children with ASD who provided artifact-free data at the outcome compared with those who did not provide data at baseline had lower ADOS Social scores $\left(F_{1,46}=5.2\right.$, $p=.03)$ and higher MSEL Verbal IQ $\left(F_{1,46}=9.9, p=.003\right)$ but did not differ in ADOS Restricted and Repetitive Behaviors $\left(F_{1,46}=1.9, p=.2\right)$ or MSEL Nonverbal IQ $\left(F_{1,46}=\right.$ $0.06, p=.8)$.

\section{EEG Protocol and Measurements}

The EEG was recorded while 140 unique color photographs of female faces (70) and toys (70) were presented in random order on a monitor. Facial images were chosen to reflect the ethnic and racial diversity of the surrounding area. Objects were common toys containing no facial features. Images were approximately $12 \mathrm{~cm}$ wide by $15 \mathrm{~cm}$ high and shown against a standard gray background ( $28 \mathrm{~cm}$ wide by $23 \mathrm{~cm}$ high).

The EEG data were recorded from 128-channel geodesic sensor nets (recorded online with reference to the vertex) at $250 \mathrm{~Hz}$, with amplification set at 1,000x, and bandpass filtering at 0.1 and $100 \mathrm{~Hz}$. Children were presented with trials consisting of 500-ms baseline, 500-ms stimulus presentation, and 1,000-ms post-stimulus recording periods followed by a random intertrial interval (0 to $500 \mathrm{~ms}$ ). Testing was terminated when the child had attended to 100 trials or was no longer attending. Trials were rejected if the child was not attending to the picture (recorded online by an observer). The EEG was filtered at $20 \mathrm{~Hz}$ and edited through an automatic artifact-detection software (Net Station 4.3.1, Electrical Geodesics, Inc, Eugene, OR) and manual editing (E.J.H.J. and S.J.W.) without knowledge of the group membership.

ERP Analyses-For the ERP analyses, the data were averaged by condition (face, object), re-referenced to the average reference, and corrected to the 100 -ms prestimulus baseline period. Electrodes with fewer than 10 included trials were interpolated from surrounding channels before re-referencing. Children with fewer than 12 artifact-free trials in each condition were excluded from the analysis. The regions and components of interest were defined with respect to the previous literature and inspection of the grand average waveform; analyses were focused on the right posterior $(97,92,86,91,85)$, left posterior 
$(58,59,60,65,66)$, and midline occipital $(73,67,78,72,77)$ electrode groupings for the P1 and N170 components and on the right anterior $(111,112,117,118,119,123,124)$, left anterior $(21,25,28,29,30,35,36)$, and midline anterior $(5,6,7,12,13,107,113)$ electrode groupings for the Nc component. Figure 1 illustrates the electrodes included in the analyses. Peaks (P1, N170, and Nc) were identified for each electrode using automatic peak detection software and verified by inspection (E.J.H.J.). Components were measured within the following time windows (from stimulus onset): P1 was 50 to $200 \mathrm{~ms}$, N170 was 150 to 350 $\mathrm{ms}$, and Nc was 200 to $700 \mathrm{~ms}$. Variables were averaged across electrode regions; all children had a minimum of three electrodes per region with visible components.

Spectral Analyses-For spectral analyses, re-referenced and baseline-corrected, artifactedited, single-trial data were divided into two 500-ms segments, corresponding to the stimulus interval and a matched-duration post-stimulus interval. Segments were de-trended and Fourier transformed using the fast Fourier transform algorithm (Matlab R2010b, MathWorks, Natick, MA). For each subject, spectral power estimates were calculated in 2$\mathrm{Hz}$ bins at each electrode in the posterior region for each segment; segments were removed from further analysis if the SD of power in the target frequency bands across an electrode group was greater than three times the mean value of that index across trials. Power estimates were averaged across trials and electrode groups (right posterior, left posterior, midline occipital electrodes) and natural log-transformed to decrease skew. Planned analyses contrasted power in the $\theta(5-7 \mathrm{~Hz})$ and a $(9-11 \mathrm{~Hz})$ bands during the presentation of face versus object stimuli, allowing the analyses to account for individual differences in absolute power.

Variables of interest were analyzed using repeated measures analyses of variance, with stimulus (face, object) and region (left, central, right) as within-subject variables, and group (ESDM, CI, and typically developing) as a between-subject variable; follow-up analyses included power ( $a$ and $\theta$ ) as a within-subject variable. Greenhouse-Geisser corrections were used. Analyses involving an interaction with group were of interest for this study. All analyses were based on the averaged lead groups (right, left, central).

\section{Results}

\section{Event-Related Potentials}

The ERP waveforms in response to faces versus objects for each group are shown in Figure 2 .

P1-P1 is a posterior positive deflection, 50 to $200 \mathrm{~ms}$ after stimulus onset, reflecting orienting to the stimulus. For P1, the significant main effects of stimulus were a larger amplitude over the central region to faces than to objects $\left(F_{2,86}=3.8, p=.03\right)$ and shorter latency responses to faces than to objects across regions $\left(F_{1,43}=5.8, p=.02\right)$. There was no main group difference in $\mathrm{P} 1$ amplitude or latency, and there were no interaction effects involving group. Adding age at testing as a covariate did not change the results.

N170-N170 is a face-sensitive posterior negative deflection 150 to $350 \mathrm{~ms}$ after stimulus onset that reflects perceptual face processing (for review, see de Haan et al. ${ }^{12}$ ). For N170, the main effects of region were found with a larger N170 amplitude at the right and left regions than at the central region $\left(F_{2,84}=7.9, p=.002\right)$ and a shorter latency at the central than at the left or right region $\left(F_{2,84}=3.7, p=.03\right)$. A main effect of stimulus showed a larger N170 negative amplitude response to faces than to objects $\left(F_{1,42}=33.0, p<.001\right)$. There were no main effects or interaction effects involving group. Adding age at testing as a covariate did not change the results. 
$\mathrm{Nc}-\mathrm{Nc}$ is a negative-going deflection that occurs around 200 to $700 \mathrm{~ms}$ after the onset of a stimulus and is recorded over the central and anterior scalp regions. ${ }^{25} \mathrm{Nc}$ is modulated by the engagement of attention to a stimulus. There was a larger (more negative) peak amplitude to objects than to faces $\left(F_{1,43}=27.7, p<0.001\right)$ that did not significantly differ by group. For Nc peak latency, there was a significant interaction between stimulus and group $\left(F_{2,43}=5.2, p=.01\right)$ and no interactions with region. As shown in Figure3, the typical and ESDM groups showed a faster Nc peak latency to faces than to objects, whereas the CI group showed a faster Nc peak latency to objects than to faces. The two groups with ASD significantly differed from each other in their patterns of Nc peak latency to faces and objects $\left(F_{1,27}=8.8, p=.006\right)$, as did the CI group and the typical group $\left(F_{1,29}=4.2, p=\right.$. $05)$. The ESDM and typical groups did not differ significantly $\left(F_{1,30}=1.7, p=.21\right)$. Adding age at testing as a covariate did not change the results. On an individual level, 11 of 15 children (73\%) in the ESDM group and 9 of 17 children (53\%) in the typical group showed a faster $\mathrm{Nc}$ response to faces than to objects compared with 4 of 14 children (29\%) in the CI group $\left(\chi^{2}=5.8, p=.05\right)$.

\section{Spectral Analysis}

Increased cortical activation was defined as decreased a power and increased $\theta$ power. ${ }^{14,26}$ There were no between-group differences in overall spectral power in the a band $\left(F_{2,43}=\right.$ $1.2, p=.32)$ or the $\theta$ band $\left(F_{2,43}=0.9, p=.40\right)$. Subsequent analyses were performed on differences between log EEG power for faces minus log EEG power for objects for $a$ and $\theta$ power. Note that negative difference scores for the a band and positive difference scores for the $\theta$ band indicate greater cortical activation for faces than for objects. Results yielded a significant interaction between power band and group $\left(F_{2,43}=6.4, p=.004\right)$. In other words, the pattern of face-object difference scores for the $\theta$ versus a band differed across groups. Specifically, as shown in Figure 4, the typical group and the ESDM group face-object difference scores showed a pattern of lower a power and greater $\theta$ power (i.e., greater activation) for faces versus objects, whereas the $\mathrm{CI}$ group showed the reverse pattern. The interaction was evident when the two groups with ASD were compared $\left(F_{1,27}=7.8, p=\right.$ $0.01)$ and when the CI group and the typical group were compared $\left(F_{1,29}=10.9, p=.003\right)$. There were no significant effects or interactions involving region.

On an individual level, 11 of 15 children (73\%) in the ESDM group and 12 of 17 children (71\%) in the typical group showed higher levels of cortical activation to faces than to objects compared with 5 of 14 children (36\%) in the CI group $\left(\chi^{2}=5.4, p=.07\right)$. The ESDM and $\mathrm{CI}$ groups were significantly different $\left(\chi^{2}=4.14, p=.04\right)$, as were the typical and CI groups $\left(\chi^{2}=3.8, p=.05\right)$, whereas the typical and ESDM groups did not differ $\left(\chi^{2}=0.03, p=\right.$. 86). Similar analyses conducted during the post-stimulus period yielded no group differences in patterns of cortical activation $(F \mathrm{~s}<1, p \mathrm{~s}>.5)$, indicating that the differences were specific to responses to the stimuli. Adding age at testing as a covariate did not change the results.

\section{Relation between Brain Activity and Primary and Secondary Behavioral Outcome Measurements}

Group Differences in Behavioral Outcomes-Children with ASD who were part of the ERP/EEG analyses in the ESDM versus CI group significantly differed in their autism symptoms, IQ, adaptive skills, and social behavior at outcome. Specifically, ADI scores for Communication Current (ESDM, mean 8.7, SD 4.9; CI, mean 13.2, SD 3.8; $F_{1,26}=7.3, p=$. 01) and ADI Social Current (ESDM, mean 9.5, SD 5.4; CI, mean 14.5, SD 6.6; $F_{1,26}=5.1, p$ $=.03$ ) were lower in the ESDM than in the CI group. Nonverbal IQ (ESDM, mean 93.1, SD 16.5; CI, mean 80.0, SD 15.8; $F_{1,27}=4.8, p=.04$ ) and Verbal IQ (ESDM, mean 95.1, SD $15.7 ; \mathrm{CI}$, mean $\left.75.1, \mathrm{SD} 18.4 ; F_{1,27}=9.9, p=.004\right)$ were higher in the ESDM than in the CI 
group. Vineland Communication (ESDM, mean 95.3, SD 15; CI, mean 76.1, SD 14.7; $F_{1,27}$ $=12.0, p=.02)$, Social (ESDM, mean 74.7, SD 10.0; CI, mean 66.5, SD 8.3; $F_{1,27}=5.7, p$ $=.02$ ), Daily Living Skills (ESDM, mean 72, SD 11.9; CI, mean 58.9, SD 7.9; $F_{1,27}=9.0, p$ $=.006$ ), and Aberrant Behaviors (ESDM, mean 76.9, SD 13.6; CI, mean 61.2, SD 7.9; $F_{1,27}$ $=14.2, p=.001$ ) were improved in the ESDM group as compared to the CI group. Social behavior assessed by the PDD-BI Expressive Social Communication composite scores (ESDM, mean 65.4, SD 6.5; CI, mean 54.5, SD 10.2; $F_{1,25}=10.3, p=.004$ ) and the PDDBI Receptive/Expression Social Communication composite scores (ESDM, mean 65.5, SD 5.6; CI, mean 55.3, SD 10.3; $F_{1,25}=9.4, p<.006$ ) were also higher in the ESDM than in the $\mathrm{CI}$ group. Moreover, children with good ERP data in the ESDM compared with the CI group (on the PDD-BI) had better Expressive Language (ESDM, mean 64.6, SD 5.8; CI, mean 54.4, SD 12.6; $F_{1,23}=6.9, p=.015$ ) and Learning, Memory, and Receptive Language (ESDM, mean 63.9, SD 3.6; CI, mean 57.6, SD 10.0; $F_{1,22}=4.6, p=.04$ ) and less Semantic Pragmatic Problems (ESDM, mean 50.4, SD 7.6; CI, mean 56.6, SD 7.4; $F_{1,22}=4.1, p=$. 055). There were no differences in ADOS scores $\left(F_{1,27}<1.0, p s>.3\right)$ between the groups at outcome.

Correlations between EEG and Behavioral Outcome-There were no significant correlations among ERP latency, EEG spectral power, and measurements of autism symptoms, IQ, language, and adaptive behavior. However, EEG measurements were correlated with levels of social behavior at outcome. On the PDD-BI, higher $\theta$ power to faces than to objects was correlated with improved PDD-BI Composite Expressive and Receptive/Expressive Social Communication Abilities $\left(r_{25}=0.51 / 0.53, p=.01 / .007\right.$, respectively), and lower a power to faces than to objects was significantly correlated with fewer social pragmatic problems $\left(r_{25}=0.44, p<.029\right)$. These data are illustrated in Figure 5 .

\section{Discussion}

Dawson et al. ${ }^{5}$ previously reported the primary behavioral outcomes of this randomized controlled trial of the ESDM intervention with young children with ASD. Children receiving the ESDM intervention demonstrated significant improvements in IQ, language, adaptive behavior, and autism diagnosis. The present article reports EEG data collected at outcome. Two types of brain activity measurements, collected in response to social (faces) versus nonsocial (toys) stimuli, were of interest. The first reflected early-stage perceptual processing of faces versus objects (N170). The second set of measurements reflected the degree of attention engagement (ERP Nc component and a power) and active cognitive processing of the stimulus ( $\theta$ power). Children receiving the ESDM and those receiving the CI did not differ from typical children in ERP measurements reflecting early-stage perceptual face processing (N170). However, EEG measurements reflecting patterns of attention engagement/active cognitive processing of social stimuli showed that children with ASD who received the ESDM intervention exhibited brain activity comparable to agematched typical children and different from children with ASD who received the CI. Typical children and children who received the ESDM intervention allotted greater attentional and cognitive resources during viewing of the social stimuli than to the nonsocial stimuli. Specifically, they exhibited a faster neural response (shorter Nc latency) when viewing faces than objects, whereas children receiving the CI showed the reverse pattern (shorter $\mathrm{Nc}$ latency to objects than to faces). Examination of individual data showed that 11 of 15 children (73\%) in the ESDM group and 9 of 17 children (53\%) in the typical group showed a faster Nc response to faces than to objects compared with 4 of 14 children (29\%) in the CI group.

Furthermore, the children with ASD who had received the ESDM intervention and typically developing children showed increased cortical activation, as reflected in decreased a power 
and increased $\theta$ power, while viewing faces compared with objects; the children receiving the $\mathrm{CI}$ showed the reverse pattern of increased activation during viewing of objects compared with faces. No group differences in cortical activation were found during the poststimulus period, suggesting that the difference in patterns of cortical activation was related to the stimuli. The $a$ and $\theta$ oscillations are generated by an interaction between glutamatergic and $\gamma$-aminobutyric acidergic neurons. Such a normalization of $\theta$ brain activity in the children who received ESDM may reflect a normalization of an imbalance between excitatory and inhibitory neurons, a hypothesized mechanism explaining neural dysfunction in autism. ${ }^{27}$

In contrast, there were no differences found between the ESDM and CI groups in the latency or amplitude of ERP measurements reflecting early orienting (P1) and early-stage perceptual processing of faces (N170). All three groups of children (ESDM, CI, and typical) showed a larger N170 amplitude to faces than to objects. Previous studies have found that toddler and preschool-age children with ASD have slower N170 and N290 (developmental precursor to N170) responses to faces than to objects. The children with ASD may have a delay in the acquisition of face expertise, reflected in atypical N170 patterns early in life, that can normalize with the later development of face expertise. One possibility is that the high levels of early intervention received by the two ASD groups were enough to allow the children to "catch up" developmentally in their perceptual face-processing abilities.

When examining only the children with good EEG data, children who received the ESDM intervention differed significantly on behavioral outcomes from those receiving the $\mathrm{CI}$ in their autism symptoms, IQ, language, adaptive behavior, and social behavior. Brain activity measurements were not correlated with diagnostic scores, IQ, language, or adaptive behavior. However, the EEG measurements were correlated with the level of social behavior at outcome. Based on the PDD-BI, it was found that greater cortical activation (lower a) while viewing faces was correlated with fewer social pragmatic problems (e.g., difficulties in reacting to the approach of others, understanding social convention, and initiating social interactions). Moreover, a greater $\theta$ power (higher activation) while viewing faces was associated with better social communication (reflecting social approach, positive affect, joint attention, use of gesture, social and imaginative play, empathy, social interaction, and imitation). Future studies will be needed to determine whether $a$ and $\theta$ power during the viewing of social versus nonsocial stimuli can serve as early efficacy biomarkers in clinical trials. Further validation of these EEG measurements by replicating their correlation with relevant clinical endpoints will be necessary.

What aspect of the ESDM intervention gives rise to the patterns of brain activity reflecting more active attention and cognitive processing of social stimuli? The ESDM model ${ }^{28}$ has a strong focus on promoting social engagement through relationship-focused activities that the child finds rewarding. It is possible that such activities, which are designed to enhance the reward value of social interactions and thereby increase attention and engagement with the social environment, contributed to the pattern of greater cortical activation when viewing social stimuli found in this study.

A limitation of the study is that approximately $60 \%$ of children with ASD were able to provide artifact-free data. Although the percentage of children with ASD with usable EEG data is comparable to other studies and did not differ as a function of baseline treatment group status, the attrition limits the ability to draw conclusions about the whole spectrum of young children with ASD. In addition, having pre- and postintervention EEG measurements would allow an examination of changes in EEG related to the intervention. Future research using larger samples is needed. 
Dawson et al. ${ }^{29}$ and others ${ }^{30}$ have suggested that decreased social attention and social motivation during the infant-toddler period have secondary consequences on brain and behavioral development of young children with ASD, exacerbating the social impairments characteristic of ASD. Early intervention that promotes attention to people and increases the motivation to engage in social interactions may serve to mitigate the emergent effects of ASD on later behavioral and brain development. As methods of earlier detection of ASD risk become available, infants with ASD will be able to benefit from very early intervention, perhaps even before the onset of the full syndrome. ${ }^{6}$ The results of the present study suggest that the ESDM intervention is associated with normalized brain activity patterns related to social attention and engagement, and that these normalized brain activity patterns are correlated with improvements in social behavior. Although early intervention is probably most optimal, a relatively brief targeted training in face processing can positively influence patterns of ERPs to faces in adults with ASD. ${ }^{31}$

In conclusion, the present study underscores the dynamic and plastic nature of early brain development in ASD and the potential of early intervention to alter the course of brain and behavioral development in young children with ASD and thereby promote the most positive long-term outcomes.

\section{Acknowledgments}

This study was supported by grant U54MH066399 from the National Institute of Mental Health (G.W.), and a postdoctoral fellowship from Autism Speaks (E.J.H.J.).

Dr. Webb served as the statistical expert for this research.

The authors gratefully acknowledge the children and families who participated in this study.

\section{References}

1. CDC. Prevalence of autism spectrum disorders-autism and developmental disabilities monitoring network, United States, 2006. MMWR Surveill Summ. 2009; 58:1-20.

2. Lord C, Risi S, DiLavore PC, Shulman C, Thurm A, Pickles A. Autism from 2 to 9 years of age. Arch Gen Psychiatry. 2006; 63:694-701. [PubMed: 16754843]

3. Luyster R, Gotham K, Guthrie W, et al. The Autism Diagnostic Observation Schedule-toddler module: a new module of a standardized diagnostic measure for autism spectrum disorders. J Autism Dev Disord. 2009; 39:1305-1320. [PubMed: 19415479]

4. Johnson CP, Myers SM. Identification and evaluation of children with autism spectrum disorders. Pediatrics. 2007; 120:1183-1215. [PubMed: 17967920]

5. Dawson G, Rogers SJ, Munson J, et al. Randomized, controlled trial of an intervention for toddlers with autism: the Early Start Denver Model. Pediatrics. 2010; 125:e17-e23. [PubMed: 19948568]

6. Dawson G. Early behavioral intervention, brain plasticity, and the prevention of autism. Dev Psychopathol. 2008; 20:775-803. [PubMed: 18606031]

7. Dawson G, Carver L, Meltzoff AN, Panagiotides H, McPartland J, Webb SJ. Neural correlates of face and object recognition in young children wiht autism spectrum disorder. Child Dev. 2002; 73:700-717. [PubMed: 12038546]

8. Webb SJ, Dawson G, Bernier R, Panagiotides H. ERP evidence of atypical face processing in young children with autism. J Autism Dev Disord. 2006; 36:881-890. [PubMed: 16897400]

9. Webb SJ, Jones E, Merkle K, et al. Developmental change inthe ERP responses to familiar faces in toddlers with autism spectrum disorders versus typical development. Child Dev. 2011; 82:18681886. [PubMed: 22004249]

10. Ackles PK, Cook KG. Event-related brain potentials and visual attention in six-month-old infants. Int J Neurosci. 2009; 119:1446-1468. [PubMed: 19922367] 
11. Moulson MC, Balas B, Nelson CA, Sinha PEEG. correlates of categorical and graded face perception. Neuropsychologia. 2011; 49:3847-3853. [PubMed: 22001852]

12. de Haan M, Johnson MH, Halit H. Development of face-sensitive event-related potentials during infancy: a review. Int J Psychophysiol. 2003; 51:45-58. [PubMed: 14629922]

13. Başar E, Başar-Eroğlu C, Karakaş S, Schürmann M. Brain oscillations in perception and memory. Int J Psychophysiol. 2000; 35:95-124. [PubMed: 10677641]

14. Klimesch WEEG. alpha and theta oscillations reflect cognitive and memory performance: a review and analysis. Brain Res Rev. 1999; 29:169-195. [PubMed: 10209231]

15. Uhlaas PJ, Haenschel C, Nikolic D, Singer W. The role of oscillations and synchrony in cortical networks and putative relevance for the pathophysiology of schizophrenia. Schizophr Bull. 2008; 34:927-943. [PubMed: 18562344]

16. Yang HH, Savostyanov AN, Tsai AC, Liou M. Face recognition in Asperger syndrome: a study on EEG spectral power changes. Neurosci Lett. 2011; 492:84-88. [PubMed: 21281694]

17. Orekhova EV, Stroganova TA, Posikera IN. Theta synchronization during sustained anticipatory attention in infants over the second half of the first year of life. Int J Psychophysiol. 1999; 32:151172. [PubMed: 10380948]

18. Orekhova EV, Stroganova TA, Posikera IN, Elam M. EEG theta rhythm in infants and preschool children. Clin Neurophysiol. 2006; 117:1047-1062. [PubMed: 16515883]

19. Lord C, Rutter M, Le Couteur A. Autism Diagnostic Interview- Revised: a revised version of a diagnostic interview for caregivers of individuals with possible pervasive developmental disorders. J Autism Dev Disord. 1994; 24:659-685. [PubMed: 7814313]

20. Lord, C.; Rutter, M.; DiLavore, P.; Risi, S. Autism Diagnostic Observation Schedule-WPS (ADOS-WPS). Los Angeles: Western Psychological Services; 1999.

21. American Psychiatric Association. Diagnostic and Statistical Manual of Mental Disorders. 4th. Washington, DC: American Psychiatric Association; p. 1994

22. Mullen, EM. Mullen Scales of Early Learning: AGS Edition. Circle Pines, MN: American Guidance Service; 1995.

23. Sparrow, S.; Balla, D.; Cicchetti, D. Vineland Adaptive Behavior Scales: Interview Edition. Circle Pines, MN: American Guidance Service; 1984.

24. Cohen IL, Schmidt-Lackner S, Romanczyk R, Sudhalter V. The PDD Behavior Inventory: a rating scale for assessing response to intervention in children with PDD. J Autism Dev Disord. 2003; 33:31-45. [PubMed: 12708578]

25. Courchesne E, Ganz L, Norcia AM. Event-related brain potentials to human faces in infants. Child Dev. 1981; 52:804-811. [PubMed: 7285651]

26. Klimesch W. Memory processes, brain oscillations and EEG synchronization. Int J Psychophysiol. 1996; 24:61-100. [PubMed: 8978436]

27. Rubenstein JL, Merzenich MM. Model of autism: Increased ratio of excitation/inhibition in key neural systems. Genes Brain Behav. 2003; 2:255-267. [PubMed: 14606691]

28. Rogers, SJ.; Dawson, G. Early Start Denver Model for Young Children with Autism: Promoting Language, Learning, and Engagement. New York: Guilford Press; 2010.

29. Dawson G, Toth K, Abbott R, et al. Early social attention impairments in autism: social orienting, joint attention, and attention to distress. Dev Psychopathol. 2004; 40:271-283.

30. Johnson MH, Griffin R, Csibra G, et al. The emergence of the social brain network: evidence from typical and atypical development. Dev Psychopathol. 2005; 17:599-619. [PubMed: 16262984]

31. Faja S, Webb SJ, Jones E, et al. The effects of face expertise training on the behavioral performance and brain activity of adults with high functioning autism spectrum disorders. J Autism Dev Disord. 2012; 42:278-293. [PubMed: 21484517] 


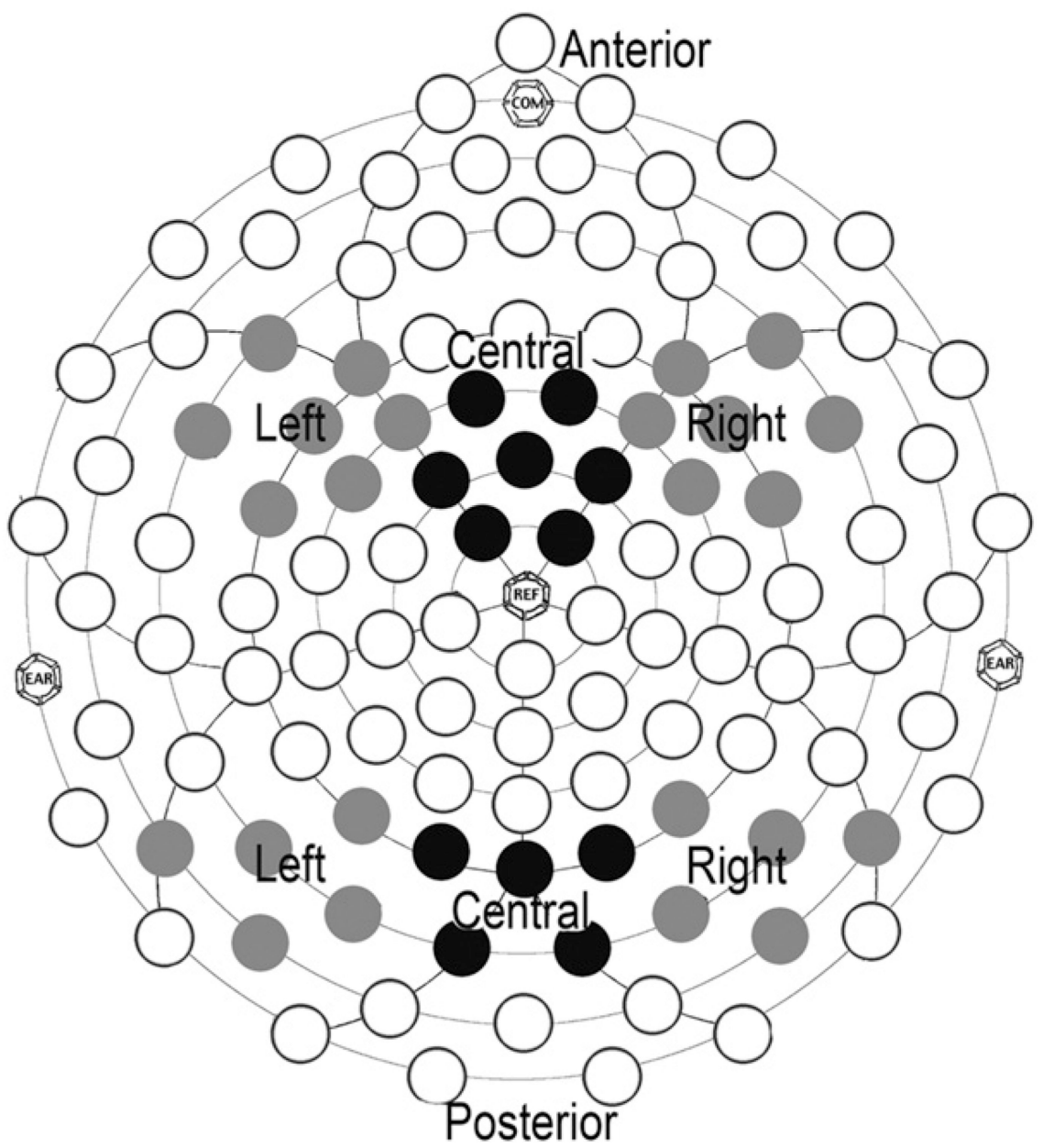

Figure 1.

Electrodes in the anterior and posterior left and right hemisphere regions (gray) and anterior and posterior central regions (black). Note: EAR, COM, and REF are anatomically marked locations for EEG sensor net placement. 

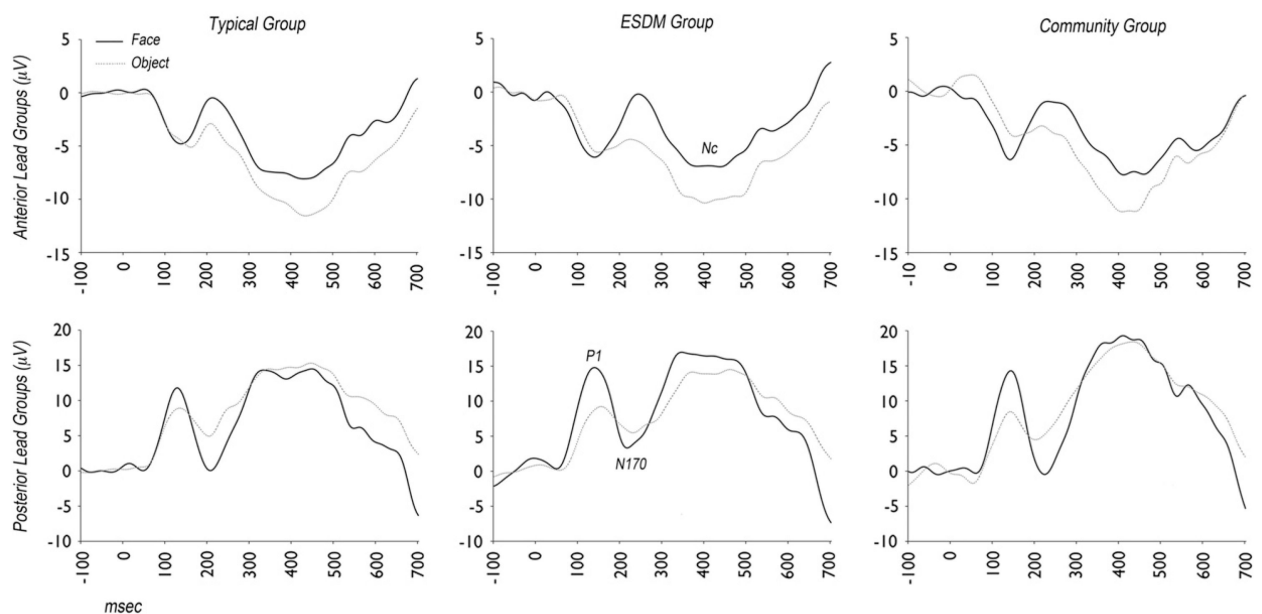

Figure 2.

Event-related potential waveforms in response to faces (black, solid) and objects (gray, dotted) from children with typical development, Early Start Denver Model (ESDM) intervention, and community intervention. 


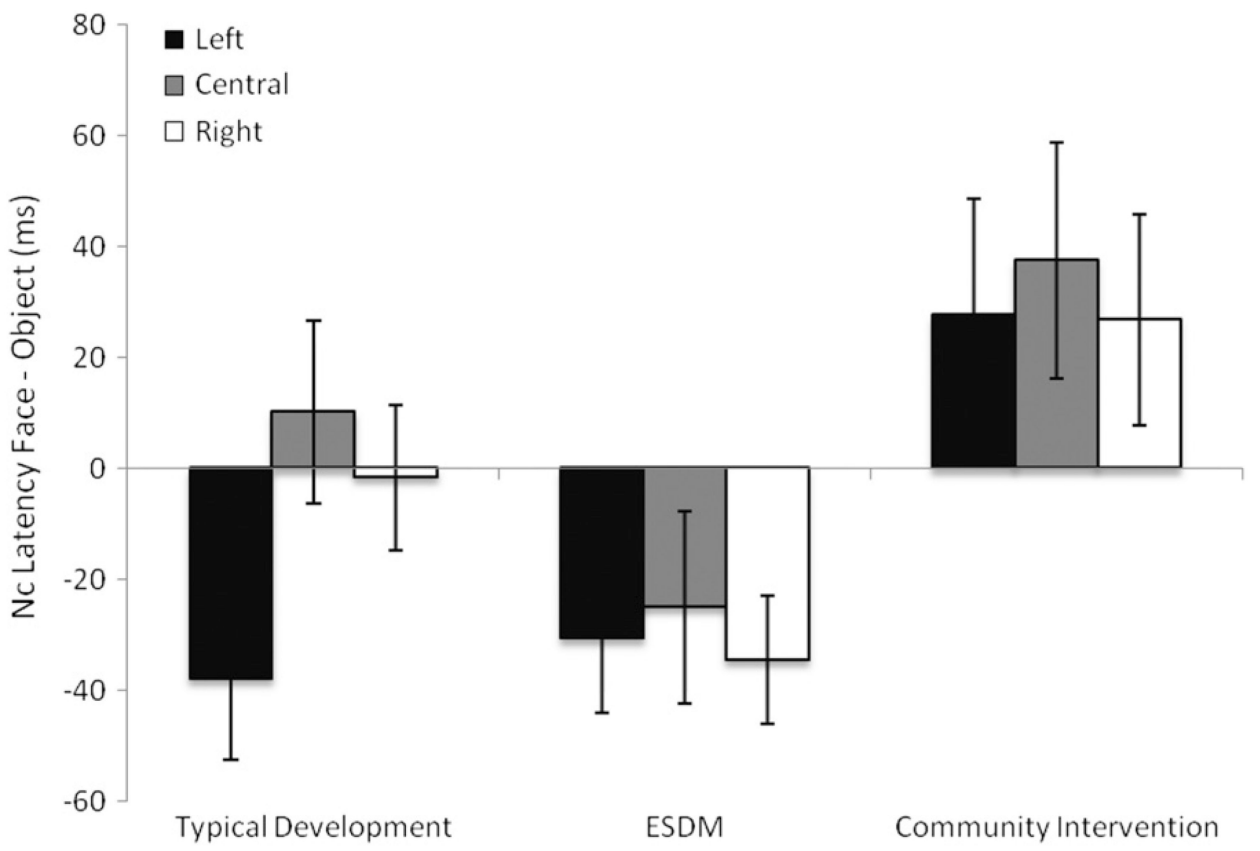

Figure 3.

Differences in peak latency of component $(\mathrm{Nc})$ responses to faces and objects for children with typical development, Early Start Denver Model (ESDM) intervention, and community intervention. Note: Negative scores represent faster responses to faces than to objects. 


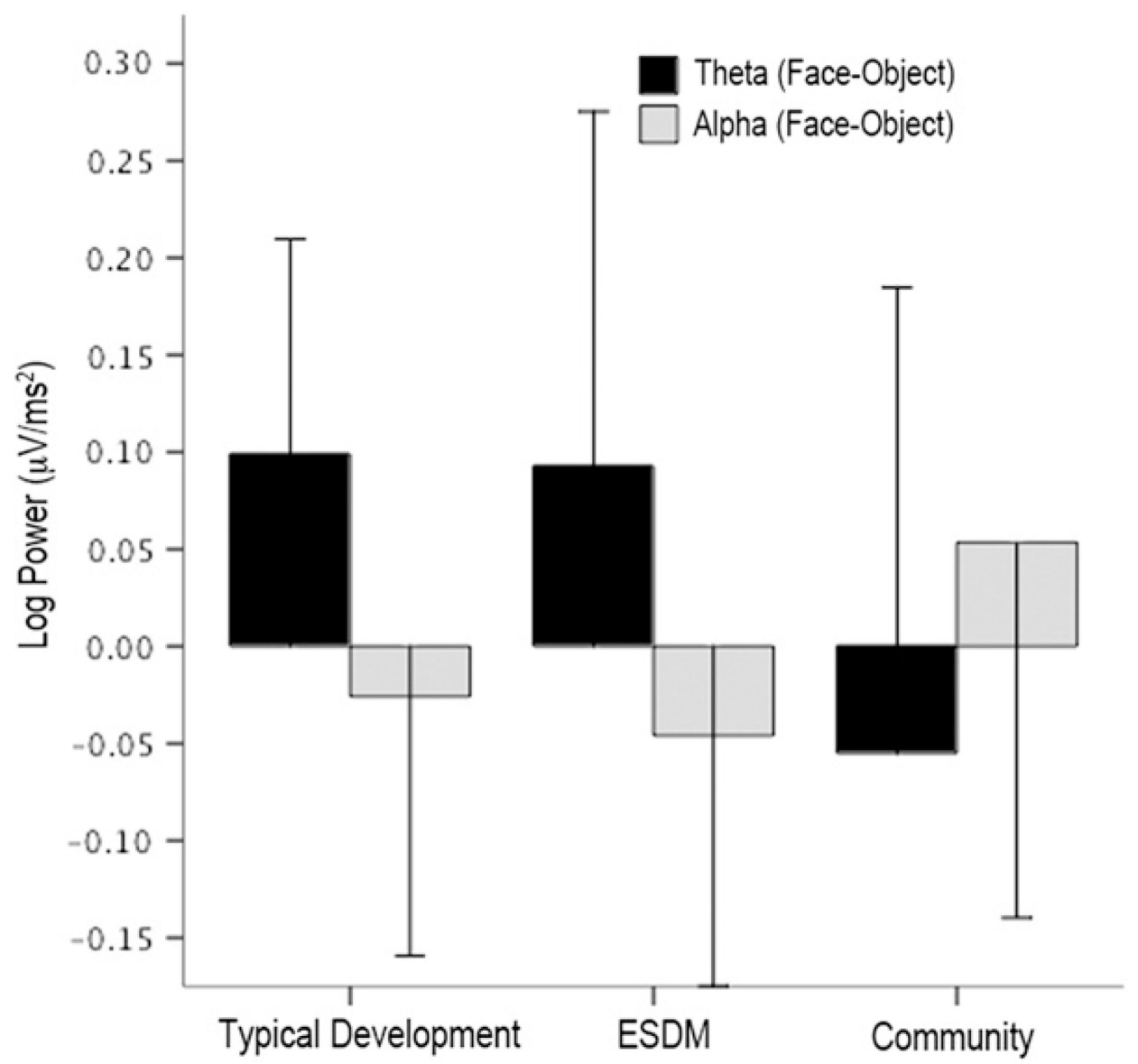

Figure 4.

Differences in patterns of brain activation in children with typical development, Early Start Denver Model (ESDM) intervention, and community intervention. Note: Dependent variables are log EEG spectral power during viewing of the faces minus objects. Positive $\theta$ and negative $a$ scores indicate greater brain activation during viewing of people's faces than of objects. 

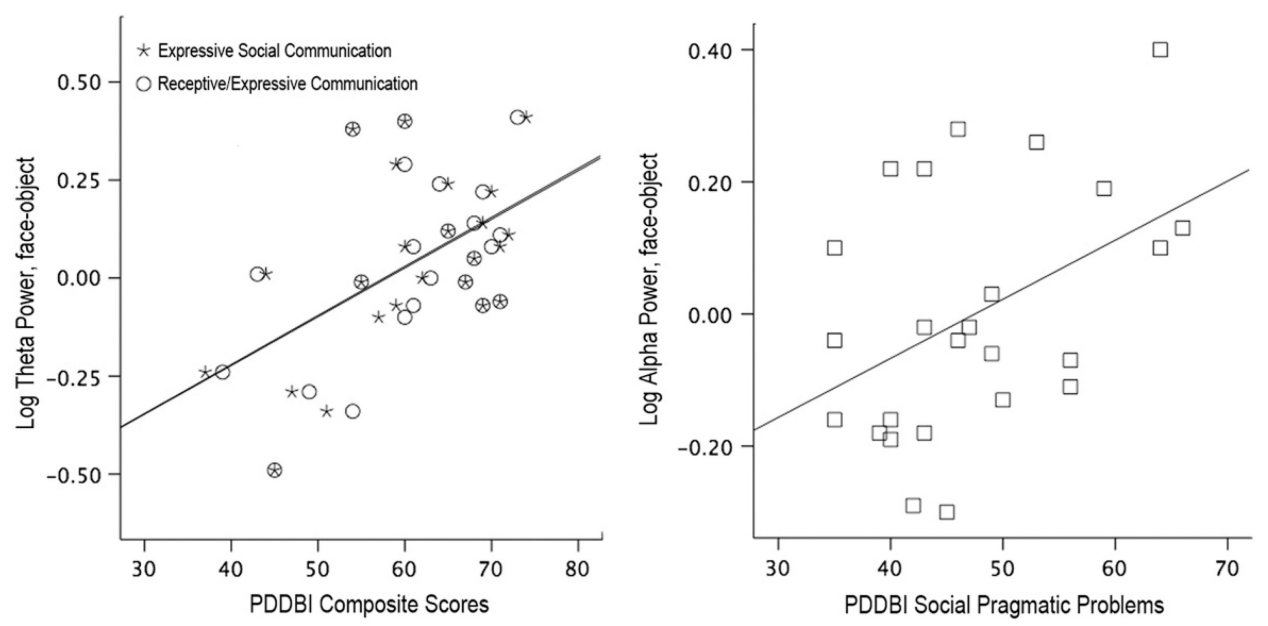

Figure 5.

(Left) Relation between $\log \theta$ power during viewing of faces versus objects and Pervasive Developmental Disorder-Behavior Inventory (PDD-BI) composite scores for Expressive Social Communication (asterisk) and Receptive/Expressive Social Communication (circles) and (right) relation between log a power during viewing of faces versus objects and the PDD-BI Social Pragmatic Problems score (squares). Note: Greater $\theta$ power difference scores indicate increased cortical activation during viewing of faces, which is associated with improved social communication scores. Lower a power difference scores indicate increased cortical activation during viewing of faces, which is associated with fewer social pragmatic problems. 